\title{
Uma Extensão Dimensional da Transformação de Lorentz e uma Aplicação à Interferência Induzida por Gravitação
}

P.H. PEREYRA, Faculdade Cenecista de Ciências e Letras de Osório - FACOS, Rua 24 de Maio 141, 95520-000 Osório, RS, Brasil.

B.E.J. BODMANN, Centro de Ciências Exatas e Tecnológicas, Universidade do Vale do Rio dos Sinos, UNISINOS, 93022-000 São Leopoldo, RS, Brasil

Resumo. No presente trabalho a unificação do espaço-tempo-massa é definida através de transformações infinitesimais penta-dimensionais. A dimensão adicional tem o papel de integrar linearmente os efeitos com origem no campo gravitacional que, entre outros, se manifestam como interferência induzida por gravitação. Uma solução consistente pela generalização dimensional da transformação de Lorentz é apresentada. Da descrição penta-dimensional espaço-tempo-massa é determinada a interferência quântica induzida por gravitação com nêutrons.

\section{Introdução}

Genericamente, teorias com espaços de referenciais diferentes de $3 \oplus 1$ são classificadas com abordagens geométricas pós-riemannianas locais e com teoria de cordas $[1,2,3,4]$. Com o presente trabalho, caracterizado pela primeira categoria citada, pretende-se elaborar a conexão entre os efeitos gravitacionais e de curvatura no espaço-tempo a partir de um espaço-tempo plano que, quando estendido através de uma dimensão adicional, se manifesta num hiperespaço plano, porém com espaço-tempo curvo. Neste espírito, a influência de uma interação é implementada aumentando-se a dimensão do espaço em consideração de uma dimensão e, portanto, surgem algumas questões:

- A dimensão adicional é apenas um método que simplifica o procedimento formal de tratar efeitos gravitacionais ou ela pode ser interpretada como válida no sentido de representar um grau de liberdade físico.

- Como do ponto de partida, é considerado um espaço-tempo plano. Deve-se perguntar se estes efeitos que curvam o espaço-tempo podem ser implementados de forma consistente, ou seja, utilizando geradores de transformações infinitesimais, que mantêm o hiperespaço plano mas encurvam a subvariedade do espaço-tempo. 
A ideia de associar efeitos massivos à quinta dimensão não é nova na literatura. Num trabalho recente a extensão mínima dimensional foi proposta interpretando a dimensão adicional como dimensão de massa $[5,6]$. Como a discussão a seguir demonstra, em relação à primeira questão a quinta dimensão descreve corretamente efeitos com origem na interferência induzida por gravitação e, com respeito à segunda questão, o presente trabalho oferece uma solução consistente pela generalização dimensional da transformação de Lorentz.

O elemento de linha invariante sob transformação de Lorentz generalizada é definido através de

$$
d \tau^{2}=d t^{2}-(d \vec{r})^{2}-d \xi^{2}
$$

onde unidades naturais são utilizadas, isto é, o intervalo de tempo é medido em unidades de comprimento tempo-luz e a última coordenada $\xi$ refere-se ao grau de liberdade induzido pela massa, conforme referência $[5,6]$. No procedimento que segue e sem restrições da generalidade define-se o elemento de linha espacial coincidente com um unico eixo $r$ (o que é valido para cenários que possuem simetria esférica), de tal modo que o sistema de referência aparece como um sistema efetivamente de dimensão $1 \oplus 1 \oplus 1$, isto é, tempo-espaço-massa. Desde que, formalmente, existem duas possibilidades de definir o elemento de linha invariante, a escolha específica do sinal na frente de $d \xi^{2}$ na equação (1.1) pode ser justificado considerando eventos ou sinais luminosos. Partículas sem massa de repouso comportam-se como partículas luminosas e, portanto, o intervalo de tempo próprio é nulo $d \tau^{2}=0$. O termo adicional $-d \xi^{2}$ no lado direito da equação (1.1) permite apenas soluções temporais, isto é, soluções massivas para a sub-variedade de espaço-tempo são válidas adotando o sinal negativo. Pelo menos formalmente este fato indica uma conexão analítica entre mundos massívos e não-massívos. Um tal cenário, consequentemente, implica em invariânca sob transformação de escala, o que, de fato, era a motivação principal na construção de geometrias relativísticas, como por exemplo o implementado nos modelos cosmológicos tipo Bianchi [5]. No presente trabalho, o objetivo é analizar transformações linearizadas sob aumento de dimensionalidade do hiper-espaço com a finalidade de investigar as suas implicações físicas.

\section{Derivação de uma Transformação de Lorentz Generalizada}

Espera-se, num dado limite da transformação de Lorentz generalizada, a redução à transformação de Lorentz comum. Adicionalmente, a derivação da generalização deve ter uma base que corresponde à base em quatro dimensões, isto é, ter como fundamentação um princípio generalizado de equivalência. Como passo inicial, a composição do espaço com dimensão cinco pode ser introduzida meramente como hipótese. Um fato aceito pela relatividade restrita é a existência de um tempo topológico, que permite a determinação local de seqüências que possuem uma conexão causal. Adicionalmente, pressupõe-se que a geometria euclidiana local é a adequada para determinar distâncias espaciais infinitesimais. Note-se que se re- 
fere à localidade no contexto generalizado ao hiper-espaço de espaço-tempo-massa. Como hipótese nova, entra uma massa topológica que permite a medição de massas ou diferênças de massa.

Para a derivação da transformação de Lorentz, são necessários os postulados já conhecidos da teoria da relatividade restrita, porém na sua forma estendida: Isotropia de espaço-massa é implementada exigindo uma relação inequívoca entre a transformação de coordenadas $\Lambda$ de um referencial para um outro e vice-versa e o seu inverso $\Lambda^{-1} \Lambda=I$. Adicionalmente, pressupõe-se a validade do princípio de relatividade. Como conseqüência, realizações de teorias massivas ou não-massivas são equivalentes desde que o princípio de equivalência generalizada implica numa quebra espontânea da invariânça de dilatação [8]. Dos postulados segue que os candidatos para as transformações são restritos aos lineares, com uma velocidade relativa constante e complexa. Assim, linhas retas em espaço-tempo-massa são transformadas em linhas retas obedecendo a regra de composição $\Lambda\left(B_{1}\right) \Lambda\left(B_{2}\right)=\Lambda\left(B_{1}+B_{2}\right)$, e, adicionalmente, coordenadas finitas em espaço-tempo-massa sempre resultam em novas coordenadas finitas. Como pré-requisito final são introduzidas simplificações convenientes, sendo elas a sincronização entre dois sistemas referenciais ao serem compatibilizados e, supondo que o ponto de tempo zero $t_{1}=t_{2}=0$ é definido no instante em que os dois referenciais coincidem, o que restringe a discussão a de uma transformação de Lorentz generalizada ao invés de a de Poincaré.

Deriva-se a transformação de Lorentz generalizada a partir de uma transformação linear infinitesimal de coordenadas. Suponha que num elemento de linha infinitesimal a velocidade generalizada seja constante. Isotropia de espaço-massa e homogenidade permitem expressar a transformação meramente pela dependência de $\beta$ e de $\eta$. Os geradores da transformação infinitesimal são

$$
G_{L}=\left(\begin{array}{ccc}
0 & -1 & 0 \\
-1 & 0 & 0 \\
0 & 0 & 0
\end{array}\right), \quad G_{M}=\left(\begin{array}{ccc}
0 & 0 & -1 \\
0 & 0 & 0 \\
-1 & 0 & 0
\end{array}\right)
$$

No limite $\eta \rightarrow 0$ ou, de forma equivalente, utilizando a "rapidez de massa" $\varepsilon \rightarrow 0$, deve resultar na transformação de Lorentz. Neste limite a quinta coordenada não se mistura com o espaço-tempo, que descreve a situação da ausência de gravitação. No limite de $\beta \rightarrow 0$ (ou $\vartheta \rightarrow 0$ ) é obtida uma transformação semelhante à de Lorentz no hiper-plano tempo-massa. Por causa dos postulados e das hipóteses anteriormente mencionados esta transformação deve ter a mesma caraterística, ou seja, a mesma estrutura que a de Lorentz. Conseqüêntemente no limite $\beta \rightarrow 0$ apenas as coordenadas de massa e o de tempo se misturam. A ultima equação que segue deriva-se explorando a simetria formal entre as coordenadas $r$ e $\xi$ e aproveitando os argumentos que foram utilizados na derivação da transformação de Lorentz, o postulado de isotropia e o princípio de relatividade.

$$
\lim _{n \rightarrow \infty}\left(I+G_{L} d \vartheta\right)^{n}=\left(\begin{array}{ccc}
\cosh \vartheta & -\sinh \vartheta & 0 \\
-\sinh \vartheta & \cosh \vartheta & 0 \\
0 & 0 & 1
\end{array}\right)
$$




$$
\lim _{n \rightarrow \infty}\left(I+G_{M} d \varepsilon\right)^{n}=\left(\begin{array}{ccc}
\cosh \varepsilon & 0 & -\sinh \varepsilon \\
0 & 1 & 0 \\
-\sinh \varepsilon & 0 & \cosh \varepsilon
\end{array}\right) .
$$

Aqui, $I$ denomina a identidade e $\vartheta$ o parâmetro de rapidez $\operatorname{com} \gamma=\cosh \vartheta$ e $\beta \gamma=$ $\sinh \vartheta$, e o análogo para tempo-massa $\varepsilon$ representando uma variação na coordenada de massa, onde $\theta=\cosh \varepsilon$ e $\eta \theta=\sinh \varepsilon$. A transformação completa é obtida utilizando o anti-commutador $\{\cdot, \cdot\}$ da transformação convencional de Lorentz e do análogo para tempo-massa.

$$
\begin{aligned}
d \Lambda & =\frac{1}{2}\left\{\left(I+G_{L} d \vartheta\right),\left(I+G_{M} d \varepsilon\right)\right\}-I \approx G_{L} d \vartheta+G_{M} d \varepsilon \\
& =\vec{G}_{C} d \vec{\alpha}=\left(\begin{array}{ccc}
0 & -d \vartheta & -d \varepsilon \\
-d \vartheta & 0 & 0 \\
-d \varepsilon & 0 & 0
\end{array}\right) .
\end{aligned}
$$

Note-se que o comutador $\left[\left(I+G_{L} d \vartheta\right),\left(I+G_{M} d \varepsilon\right)\right]-I \equiv 0$ correspondente à transformação espaço-tempo-massa é nulo. Qualquer transformação finita pode ser construida através de uma aplicação sucessiva das transformações infinitesimais, o que resulta em

$$
\begin{aligned}
\Lambda & =\lim _{n \rightarrow \infty}(I+d \Lambda)^{n}=\lim _{n \rightarrow \infty}\left(I+\vec{G}_{C} d \vec{\alpha}\right)^{n} \\
& =\left(\begin{array}{ccc}
\cosh |\alpha| & -\frac{\vartheta}{|\alpha|} \sinh |\alpha| & -\frac{\varepsilon}{|\alpha|} \sinh |\alpha| \\
-\frac{\vartheta}{|\alpha|} \sinh |\alpha| & \frac{\varepsilon^{2}+\vartheta^{2} \cosh |\alpha|}{|\alpha|^{2}} & \frac{\vartheta \varepsilon}{|\alpha|^{2}}(\cosh |\alpha|-1) \\
-\frac{\varepsilon}{|\alpha|} \sinh |\alpha| & \frac{\vartheta \varepsilon}{|\alpha|^{2}}(\cosh |\alpha|-1) & \frac{\vartheta^{2}+\varepsilon^{2} \cosh |\alpha|}{|\alpha|^{2}}
\end{array}\right)
\end{aligned}
$$

e onde foi introduzida a rapidez complexa $\alpha$ com $\Re e\{\alpha\}=\vartheta$ e $\Im m\{\alpha\}=\varepsilon$. A partir dessa expressão verifica-se diretamente que nos respectivos limites $\varepsilon \rightarrow 0$ e $\vartheta \rightarrow 0$ as transformações finitas de Lorentz e uma transformação pura de tempo-massa são recuperadas. Note que um argumento complexo como rapidez generalizada está de acordo com o que se espera intuitivamente de uma geometria euclidiana no espaço-massa por causa da sua conexão através de rotações ordinárias.

\section{Efeitos Relativísticos no Contínuo Espaço- Tempo-Massa}

\subsection{Efeitos Relativísticos de Espaço-Tempo-Massa}

Os três efeitos relativísticos relevantes nas cinco dimensões são, dilatação de tempo, contração de comprimento e, por causa da presença da quinta dimensão, "contração de massa",

$$
d t_{2}=\cosh |\alpha| d t_{1},
$$




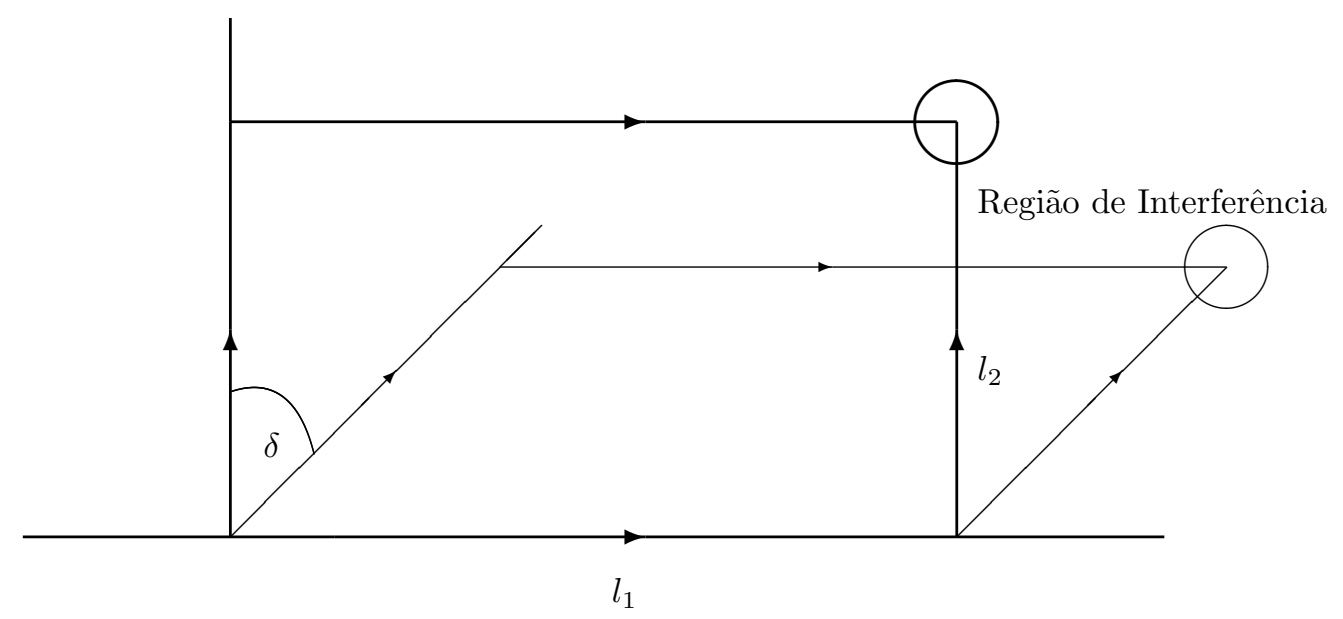

Figura 1: Interferometria com partículas para a demonstração da interferência quântica induzida por gravitação de acordo com referência [7].

$$
\begin{aligned}
d r_{2} & =\frac{\Im m^{2}\{\alpha\}+\Re e^{2}\{\alpha\} \cosh |\alpha|}{|\alpha|^{2}} d r_{1}, \\
d \xi_{2} & =\frac{\Re e^{2}\{\alpha\}+\Im m^{2}\{\alpha\} \cosh |\alpha|}{|\alpha|^{2}} d \xi_{1} .
\end{aligned}
$$

Verifica-se que, para pequenos valores de rapidez $\vartheta$ e $\varepsilon$, os efeitos espaciais desacoplam dos efeitos de massa e a influência principal é relacionada ao tempo. Caso o modelo presente represente alguma realidade física, são experiências envolvendo medição de tempo que são adequadas para verificar estes efeitos, que justifica a abordagem de combinar uma transformação generalizada através dos constituintes representando contribuições de espaço-tempo e de tempo-massa.

\subsection{Interferência Quântica Induzida por Gravitação}

Existem experiências que mostram como efeitos gravitacionais, mesmo com ordem de grandeza pequena, são comensuráveis através de interferência quântica induzida por gravitação. Em seguida, será brevemente descrita uma experiência [7] cujo resultado será interpretado em termos da descrição penta-dimensional espaço-tempomassa. Nesta experiência, um feixe monoenergético de partículas (na experiência de referência [7] trata-se de nêutrons) passa através de um monocristal fino. Este tem o papel de dividir o feixe incidente em dois feixes cada um com direção de propagação diferente da outra. Após de uma certa distância os dois feixes passam novamente através de um monocristal que redireciona uma parte de cada feixe numa região comum (o plano de observação) onde estes se superpõem para dar origem à interferência (veja Figura 1). Um dos feixes segundários percorre primeiro a distância $l_{1}$ e depois $l_{2}$ enquanto o segundo feixe percorre as distâncias na ordem 
invertida. Supondo que, inicialmente, o plano definido pelo percurso dos feixes está no mesmo equipotencial quando o plano é girado de um ângulo $\delta$ em torno de um eixo que coincide com a direção de $l_{1}$, conforme Figura 1 , as partes das trajetórias correspondentes ao longo de $l_{1}$ não têm mais o mesmo potencial para os dois feixes. Consequentemente, a energia potencial do feixe dois aumenta por $\Delta E=g l_{2} \sin \delta$ em comparaçao ao feixe um. Portanto, a defasagem na descrição penta-dimensional se relaciona à diferença de energia quando os termos dominantes são levados em conta

$$
\Delta \phi=\frac{\Delta E t}{\hbar}=\frac{\Delta E m \lambda l_{1}}{2 \pi \hbar^{2}} .
$$

A fim de reproduzir as observações calcula-se o transporte de energia em torno do quadrado no sentido de um transporte paralelo, utilizando a transformação $E_{i}=$ $\cosh \left(\sqrt{\varepsilon_{i}^{2}}\right) E_{0}$, onde $E_{i}$ refere-se às energias em relação ao referencial fracamente acelerado e $E_{0}$ é a energia em relação referencial inercial, que tem como diferença de fase

$$
\Delta \phi=\frac{1}{2} \frac{\left(\varepsilon_{1}^{2}-\varepsilon_{2}^{2}\right) m^{2} c^{2} \lambda l_{1}}{2 \pi \hbar^{2}} .
$$

Interpretando $1 / 2\left(\varepsilon_{1}^{2}-\varepsilon_{2}^{2}\right) c^{2}=g l_{2} \sin \delta$ como sendo a diferença pelo potencial gravitacional, a diferença de fase em termos de um ângulo de rotação é

$$
\Delta \phi=\frac{m^{2} l_{1} l_{2} \lambda g \sin \delta}{2 \pi \hbar^{2}},
$$

o que coincide com o resultado experimental da referência [7]. A Figura 2 mostra as modulações teóricas esperadas para um fluxo constante de nêutrons, devido à interferência quântica induzida por gravitação. Observe-se que a interpretação não resultou em nenhuma contradição. A quinta dimensão se tornou útil no tratamento de efeitos gravitacionais no nível microscópico como por exemplo da interferência quântica induzida pela gravitação.

\section{Conclusões}

A implementação e a derivação da geometria através de transformações infinitesimais permitem recuperar a teoria da relatividade restrita no limite da quinta coordenada nula e, portanto, mantêm válida esta teoria que já passou por uma série de testes experimentais. A transformação finita entre referenciais inerciais em cinco dimensões é o resultado médio de um transporte paralelo (anti-comutação), enquanto o comutador é nulo, que justifica o termo de um referencial inercial generalizado. Nessa definição são permitidas acelerações que provavelmente devem obedecer a uma equação dinâmica que podem ser até quânticas. Como primeiro passo foram abordados efeitos gravitacionais tratando de configurações que possuem curvatura pela sub-variedade no espaço-tempo.

A escolha da geometria específica espaço-massa parece que exige teorias sem massa por causa da sua propriedade de separar passados e futuros de partículas massivas em cinco dimensões e, quando preserva causalidade, deve descartar soluções 


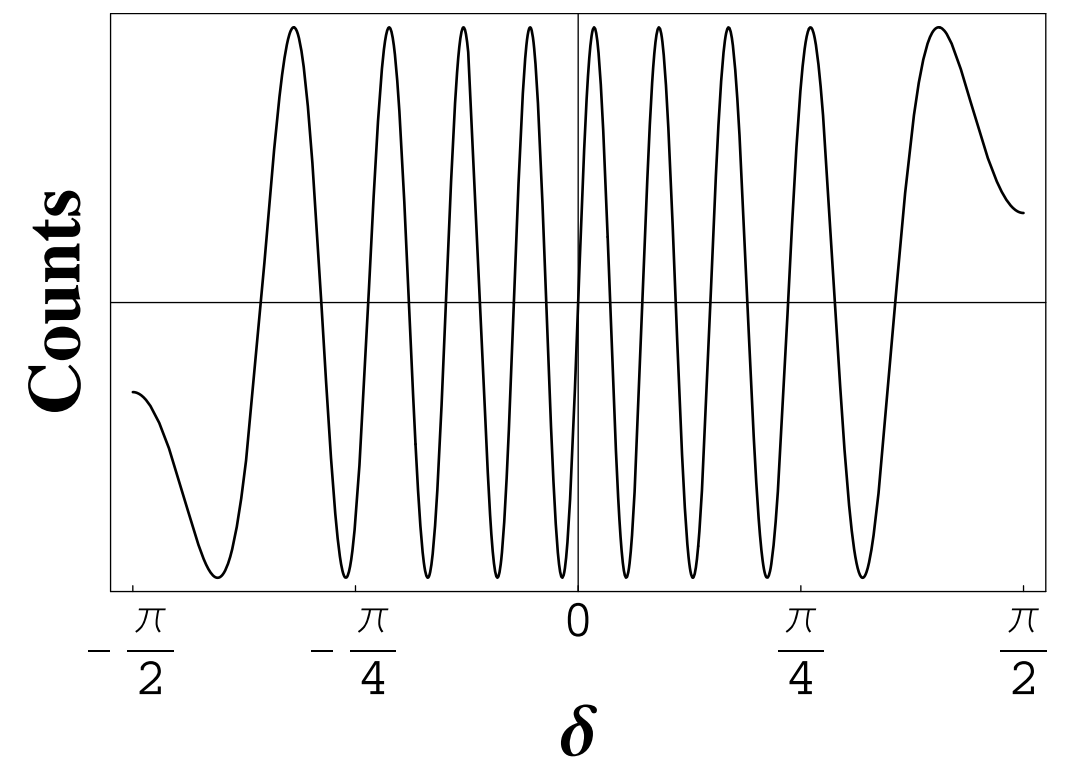

Figura 2: Predição teórica de interferência quântica induzida por gravitação para diferentes ângulos de rotação na descrição penta-dimensional, espaço-tempo-massa.

massivas. Para partículas sem massa, este problema não existe pois a quinta dimensão permite uma conexão analítica entre mundos sem massa de repouso e mundos massivos. A dimensão massiva foi introduzida via meio topológico, portanto, "produção de massa" deve se basear em uma álgebra que deixa invariante o cone luz penta-dimensional e define rotações no hiper-plano espaço-massa. A mesma questão foi abordada partindo de um Lagrangiano Dirac-Maxwell, pois os detalhes do mecanismo para produção de massa eram impostos como uma hipótese [8]. No trabalho presente a hipótese da quebra espontânea da dilatação como gerador para mundos massivos pode ser abandonada porém pressupondo a validade do princípio de relatividade generalizada. Conseqüentemente realizações de teorias massivas ou não-massivas são equivalentes, desde que o princípio de equivalência generalizada implica numa quebra espontânea da invariância de escala.

\footnotetext{
Abstract. In the present work a unification of space-time-mass is defined by an infinitesimal transformation in five dimensions. The additional dimension is supposed to integrate in a linear fashion effects with origin in the gravitational field, which among others give rise to gravity induced interference. A consistent solution for the dimensional generalization of the Lorentz transform is presented. From the five dimensional space-time-mass description gravity induced quantum interference of neutrons is determined.
} 


\section{Referências}

[1] M. Kaku, "Quantum Field Theory", Oxford University Press, New York, Oxford, 1993.

[2] M. Nakahara, "Geometry, Topology and Physics", Institute of Physics Publishing, Bristol, Philadelphia, 1990.

[3] A.N. Schellekens, "Introduction to String Theory", Preprint NIKHEF-H/99999, NIKHEF, Amsterdã, 1999.

[4] G. Veneziano, Quantum geometric origin of all forces in string theory, em "The Geometric Universe" (S.A. Hugget, L.J. Mason, K.P. Tod, S.T. Tsou, N.M.J. Woodhouse, eds.), Oxford University Press, New York, Oxford, 1998.

[5] P.H. Pereyra, "Soluções não Planas no Modelo Cosmológico Bianchi tipo V na Teoria 5d-Espaço-Tempo-Massa", Dissertação de Mestrado, Universidade Federal do Rio Grande do Sul, Instituto de Matemática, Porto Alegre, RS, 1999.

[6] J.M. Overduin, P.S. Wesson, "Kaluza-Klein Gavity", Preprint gr-qc/9805018 Stanford University, 1998.

[7] R. Collela, A. Overhauser, S. Werner, Phys. Rev. Lett., 34 (1975), 1472.

[8] B.E.J. Bodmann, Th.A.J. Maris, Phys Lett. B, 495 (2000), 98. 